\title{
Reviewer Recognition
}

๑ International Spinal Cord Society 2018

We would like to thank all those listed below for taking the time to review for Spinal Cord Series and Cases in 2017-your generosity is much appreciated and we hope your association with the journal continues in the future.

\author{
Afzal, Suhail \\ Aisen, Mindy \\ Aito, Sergio \\ Akbar, Michael \\ Alessio, Helaine \\ Alexander, Craig \\ Annie, Philip \\ Anwar, Fahim \\ Arora, Mohit \\ Baba, Hisatoshi \\ Bakhsh, Ahmed \\ Baumberger, Michael \\ Berbrayer, David \\ Bickel, C Scott \\ Biering-Sørensen, Fin \\ Birch, Brian \\ Blockmans, Inge \\ Bryce, Thomas \\ Cecchi, Paolo \\ Cho, Stephanie \\ Couch, James \\ Del Popolo, Giulio \\ DeVivo, Michael \\ Dionyssiotis, Yannis \\ Ditunno Jr., John \\ Donovan, William \\ El Masri, Wagih
}

Elliott, Stacy
Everhart Skeels, Sarah
Field-Fote, Edelle
Francesco, Scaravilli
Frankel, DR
Fried, Guy
Fujimori, Takahito
Furuya, Takeo
Georgiou, Margarita
Goh, Khean-Jin
Graves, Daniel
Gustafson, Kristen
Habek, Mario
Hagen, Ellen Merete
Hanna-Mitchell, Ann
Hasnan, Nazirah
Hill, Vernon
Ho, Chester
Houlihan, Bethlynn
Irgens, Ingebjorg
Kasch, Helge
Katoh, Shinsuke
Kennelly, Michael
Kim, Ki-Jeong
Konstantinidis, Charalampos
Krassioukov, Andrei
Kyriakides, Athanasios

Lavelle, John

Lee, Michael

Levi, Richard

Lieberman, Jesse

Liu, Nan

Lude, Peter

Mahajan, Rajat

Marino, Ralph

Marson, Lesley

Meves, Robert

Middleton, James

Morse, Leslie

Murillo, Narda

Murray, $\mathrm{H}$.

Nair, Krishnan

Nance, Patricia

New, Peter

Nulle, Anda

Oliviero, Antonio

Post, Marcel

Previnaire, Jean

Pyrgeli, Maria

Raasck, Kyle

Rainer, Abel

Ramos, Edwardo

Rutkowski, Susan

Sabre, Liis
Sakakibara, Brodie

Savic, Gordana

Scheel-Sailer, Anke

Sliwinski, Martha

Slocum, Chloe

Smith, Andrew

Suchdev, Kushak

Taira, Takaomi

Taylor, Andrew

Tederko, Piotr

Teixeira, William

Teles, Alisson

Tepper, Mitch

Tharion, George

Thomas, George

Tominaga, Hiroyuki

Trbovich, Michelle

Urdzikova, Lucia

Vogel, Larry

Waters, Robert

Wecht, Jill

Welling, Karen-Lise

Wen, Wendy

Werhagen, Lars

Willis, Mary

Wing, Peter

Wirz, Markus 